\title{
On the Concept of Limit Inferior and Limit Superior
}

\author{
M. Seyyit Seyyidoğlu' ${ }^{1}$ N. Özkan Tan²* \\ ${ }^{1}$ Uşak University, Faculty of Science and Letters, Department of Mathematics, 1 Eylül Campus, Uşak, Turkey \\ seyyit.seyyidoglu@usak.edu.tr \\ ${ }^{2}$ Uşak University, Faculty of Science and Letters, Department of Mathematics, 1 Eylül Campus, Uşak, Turkey \\ ${ }^{*}$ Corresponding author \\ Recieved: $15^{\text {th }}$ June 2016 \\ Accepted: $1^{\text {st }}$ January 2017 \\ DOI: $10.18466 /$ cbayarfbe. 302763
}

\begin{abstract}
This paper is concerned with the giving a generalization of statistically limit inferior and statistically limit superior defined in [15]. Properties of $\Delta$ - $\limsup _{t \rightarrow \infty} f(t)$ and $\Delta-\liminf _{t \rightarrow \infty} f(t)$ is given for a function defined on time scale $\mathbb{T}$.
\end{abstract}

Keywords - Statistical limit, Cluster point, Limit point, $\Delta$-convergence.

\section{Introduction}

The theory of statistical convergence has been introduced in [1]. This concept become useful tool for some fundamental subjects of mathematics the last half of the century such as number theory [4], [5], trigono-metric series [6], summability theory [7], measure theory [8], optimization theory [9] and approximation theory [10]. Fridy progressed with the concept of statistically Cauchy sequence in [2] and proved that it is equivalent to statistical convergence. Besides in [3], the notion of the statistical limit point is defined by him.

The theory of time scales was first constructed by Hilger in his Ph. D. thesis in [11]. The concept of time scale is based on the aspect of unite discrete analysis and continuous analysis. The time scale $\mathbb{T}$ is an arbit-rary nonempty closed subset of the real numbers $\mathbb{R}$. In fact, $\mathbb{T}$ is a complete metric space with the usual met-ric. Throughout this paper we consider a time scale $\mathbb{T}$ with the topology that inherits from the real numbers with the standart topology. For detailed information about time scale theory, one can see [12] and [13]. Measure theory on time scales has been introduced in [16], then further studies were made by in [17] and [18]. DenizUfuktepe defined Lebesgue-Stieltjes $\Delta$ and $\nabla$ measures and by using these measures they defined an integral which is adaptable to the time scale, specifically Lebesgue-Stieltjes $\Delta$-integral, in [19]. In the light of these studies, let us introduce some time scale and measure theoretic notations. The forward jump operator $\sigma: \mathbb{T} \rightarrow \mathbb{T}$ for each $t \in \mathbb{T}$ by via formula,

$$
\sigma(t):=\inf \{s \in \mathbb{T}: s>t\}
$$

For $a, b \in \mathbb{T}$ with $a \leq b$ we define the interval $[a, b]$ in $\mathbb{T}$ by

$$
[a, b]=\{t \in \mathbb{T}: a \leq t \leq b\} .
$$

Open intervals and half-open intervals are defined similarly. Let $\mathcal{S}$ be semiring of left-closed and right-open intervals and $m^{*}$ be Caratheodory extension of the Lebesgue set function $m$ which is defined by $m([a, b))=b-a$, associated with the family $\mathcal{S}$ in the time scale $\mathbb{T}$ as in the real case. Also let $\mathfrak{M}\left(m^{*}\right)$ be the $\sigma$-algebra of all $m^{*}$ measurable sets. Recall that $\mathfrak{M}\left(m^{*}\right)$ consists of such a subset $E$ has the property that $m^{*}(A)=$ $m^{*}(A \cap E)+m^{*}\left(A \cap E^{c}\right)$ for all $A \subset \mathbb{T}$. It is well known that the restriction of $m^{*}$ to $\mathfrak{M}\left(m^{*}\right)$ which we denote by $\mu_{\Delta}$ is a countably additive measu-re 
on $\mathfrak{M}\left(m^{*}\right)$. This measure called Lebesgue $\Delta$ measure. The measurable subsets of $\mathbb{T}$ is called $\Delta$ measurable and a function $f: \mathbb{T} \rightarrow \mathbb{R}$ is called $\Delta$ measu-rable function, if $f^{-1}(\mathcal{O}) \in \mathfrak{M}\left(m^{*}\right)$ for every open subsets $\mathcal{O}$ of $\mathbb{R}$. From [16] we know that if $a, b \in \mathbb{T}$ and $a \leq b$ then

$$
\mu_{\Delta}([a, b))=b-a, \mu_{\Delta}((a, b))=b-\sigma(a)
$$

If $a, b \in \mathbb{T}-\{\max \mathbb{T}\}$ and $a \leq b$ then

$$
\mu_{\Delta}((a, b])=\sigma(b)-\sigma(a), \mu_{\Delta}([a, b])=\sigma(b)-a .
$$

In [14], the concept of $\Delta$-density which is generalization of the of concept natural density by using measu-re theoretic approach is given. If $A$ is a $\Delta$ measurable subset of $\mathbb{T}$ and $a=\min \mathbb{T}$, the $\Delta$ density of $A$ in $\mathbb{T}$ is defined by

$$
\lim _{s \rightarrow \infty} \frac{\mu_{\Delta}(A(s))}{\sigma(s)-a}
$$

(if this limit exists) where $A(s)=\{t \in A: t \leq s\}$. The $\Delta$-density function can be considered as a probabilistic finite additive measure on the algebra of subset of $\mathbb{T}$ which have a $\Delta$-density. By using the $\Delta$ density we obtained a new type of convergence which is generalization of the natural statistical convergence and statistical Cauchy sequences definitions. In [20],

the concepts of the $\Delta$-limit and the $\Delta$-cluster point are given. These concepts are generalization of the concept of the statistical limit and statistical cluster point defining in [3]. Let us remember some of these notions. A $\Delta$-measurable function $f$ is called $\Delta$-convergent to the number $L$ if

$$
\delta_{\Delta}\left(f^{-1}((L-s, L+s))\right)=1
$$

for all $s>0$. A measurable set $K$ is called $\Delta$-non thin subset of $\mathbb{T}$ if it may have a positive $\Delta$-density or may not have even a $\Delta$-density and a measurable set $K$ is called a $\Delta$-null subset of $\mathbb{T}$ if $\delta_{\Delta}(K)=0$. A measurable function $f: \mathbb{T} \rightarrow \mathbb{R}$ is called $\Delta$-bounded if there exists a real number $r$ such that $\delta_{\Delta}(\{t \in$ $\mathbb{T}:|f(t)| \leq r\})=1$. The number $L$ is called $\Delta$-cluster point of a measurable function $f$ if $\delta_{\Delta}\left(f^{-1}((L-\right.$ $s, L+s))$ ) is a $\Delta$-non thin subset of $\mathbb{T}$. We will use the symbol $\Gamma_{f}$ to denote all $\Delta$-cluster points of a $\Delta$ measurable function $f$. The set $\Gamma_{f}$ is closed subset of $\mathbb{T}$.

The main purpose of the present paper is to extend the notions of statistical limit inferior and statistical limit superior point defined in [15] by using real valued functions defined on time scale.

\section{$2 \Delta$-limit superior and $\Delta$-limit inferior}

In this section, we introduce the notion of $\Delta$-limit superior a $\Delta$-limit inferior for a $\Delta$-measurable function defined on $\mathbb{T}$. We will further with properties of these concepts and we will give the some relations with the $\Delta$-cluster points defined in [20] and classical limit inferior and limit superior points concepts.

Definition 2.1 Let $f: \mathbb{T} \rightarrow \mathbb{R}$ be a $\Delta$-measurable function. If we consider the following subsets of :

$$
\begin{gathered}
A(f):=\left\{y \in \mathbb{R}: f^{-1}((-\infty, y)) \text { is a } \Delta \text {-non thin set }\right\} \\
B(f):=\left\{y \in \mathbb{R}: f^{-1}((y, \infty)) \text { is a } \Delta \text {-non thin set }\right\}
\end{gathered}
$$

Then the following extended real number

$$
\Delta-\limsup _{t \rightarrow \infty} f(t):=\sup B(f)
$$

is called $\Delta$-limit supreior of the function $f$ whenever $t \rightarrow \infty$. Similarly follwing extended number

$$
\Delta-\liminf _{t \rightarrow \infty} f(t):=\inf A(f)
$$

is called $\Delta$-limit inferior of the function $f$ whenever $t \rightarrow \infty$. Let us start with the expecting property of $\Delta$ - $\limsup f(t)$ and $\Delta-\liminf f(t)$.

Proposition 2.2 Let $f: \mathbb{T} \rightarrow \mathbb{R}$ be a $\Delta$-measurable function. Then we have

$$
\Delta-\limsup _{t \rightarrow \infty} f(t)=-\Delta \text {-liminf }_{t \rightarrow \infty}(-f(t)) .
$$

Proof From definition of $A(f)$ and $B(f)$ we have,

$$
A(-f)=\left\{y \in \mathbb{R}: \bar{\delta}_{\Delta}(\{t \in \mathbb{T}: f(t)>-y\})>0\right\}
$$

and

$$
\begin{aligned}
-A(-f)= & \left\{-y \in \mathbb{R}: \bar{\delta}_{\Delta}(\{t \in \mathbb{T}: f(t)>-y\})>0\right\} \\
= & \left\{y \in \mathbb{R}: \bar{\delta}_{\Delta}(\{t \in \mathbb{T}: f(t)>y\})>0\right\} \\
& =B(f) .
\end{aligned}
$$

So that we have,

$$
-\sup B(f)=-\sup (-A(-f))=\inf A(-f) .
$$

Desired equality is easily obtained from above equality.

The following two theorems tell us a necessary and sufficient condition for being a finite valued $\Delta$-limit supreior point and $\Delta$-limit inferior point of a function defined on time scale $\mathbb{T}$. 
Theorem 2.3 Let $f: \mathbb{T} \rightarrow \mathbb{R}$ be a $\Delta$-measurable function. The real number $L$ is $\Delta$-limit supreior point of the function $f$ if and only if for all $s>0$,

i) $f^{-1}((L-s, \infty))$ is a $\Delta$-non thin subset of $\mathbb{T}$,

ii) $f^{-1}((L+s, \infty))$ is a $\Delta$-null subset of $\mathbb{T}$.

Proof We will show that (i) and (ii) hold for all

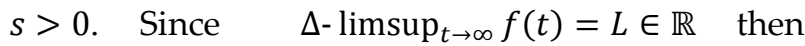
$B(f) \neq \emptyset$. From sup properties of real numbers, for all $s>0$ there exists $y \in B(f)$ such that $L-s<y$. Since $\quad f^{-1}((y, \infty)) \subset f^{-1}((L-s, \infty))$ and $f^{-1}((y, \infty))$ is a $\Delta$-non thin subset of $\mathbb{T}$ then $f^{-1}((L-s, \infty))$ is a $\Delta$-non thin subset of $\mathbb{T}$. Now assume that (ii) does not hold. Then there exists $s>0$ such that $f^{-1}((L+s, \infty))$ is a $\Delta$-non thin subset of $\mathbb{T}$. That means $L+s \in B(f)$. This contrdicts with $L=\sup B(f)$. Therefore (i) and (ii) hold for all $s>0$. Now we will show that $\Delta$ - $\limsup _{t \rightarrow \infty} f(t)=L \in \mathbb{R}$. From (ii) the real number $L$ is an upper bound of $B(f)$. If $M$ is another upper bound of $B(f)$ then from (i) it should be greater than or equal to $L$. So that $\sup B(f)=L$.

Theorem 2.4 Let $f: \mathbb{T} \rightarrow \mathbb{R}$ be a $\Delta$-measurable function. The real number $L$ is $\Delta$-limit inferior point of the function $f$ if and only if for all $s>0$,

i) $f^{-1}((-\infty, L+s))$ is a $\Delta$-non thin subset of $\mathbb{T}$,

ii) $f^{-1}((-\infty, L-s))$ is a $\Delta$-null subset of $\mathbb{T}$.

Proof It is easily obtained from Proposition 2.2 and Theorem 2.3.

Theorem 2.5 Let $f: \mathbb{T} \rightarrow \mathbb{R}$ be a $\Delta$-measurable function. The real number $L$ is $\Delta$-limit supreior of the function $f$ if and only if $=\sup \Gamma_{f}$.

Proof We will show that $\sup B(f)=\sup \Gamma_{f}$. If $\Gamma_{f}$ is an unbounded subset of $\mathbb{R}$ then the set $B(f)$ is also unbounded then equality holds. Now let define $\sup \Gamma_{f}=L_{1}$ and $\sup B(f)=L_{2}$ then since the real number $L_{2}$ is the $\Delta$-limit supreior of the function $f$, from Theorem 2.3-(i), $f^{-1}\left(\left(L_{2}-s, \infty\right)\right)$ is a $\Delta$-non thin and $f^{-1}\left(\left(L_{2}+s, \infty\right)\right)$ is a $\Delta$-null subset of $\mathbb{T}$. If we subtract a $\Delta$-null set from a $\Delta$-non thin set then we obtain a $\Delta$-non thin set. Therefore

$$
\begin{array}{r}
f^{-1}\left(\left(L_{2}-s, L_{2}+s\right)\right)=f^{-1}\left(\left(L_{2}-s, \infty\right)\right) \\
-f^{-1}\left(\left[L_{2}+s, \infty\right)\right)
\end{array}
$$

is a $\Delta$-non thin subset of $\mathbb{T}$. So that $L_{2}$ is a $\Delta$-cluster point of $f$ and we have $L_{1} \geq L_{2}$. Now we assume that $L_{1}>L_{2}$ and $s_{1}:=L_{1}-L_{2}>0$. Since

$$
f^{-1}\left(\left(L_{1}-\frac{s_{1}}{2}, L_{1}+\frac{s_{1}}{2}\right)\right) \subset f^{-1}\left(\left(L_{2}+\frac{s_{1}}{2}, \infty\right)\right)
$$

and Theorem 2.3 (ii), $f^{-1}\left(\left(L_{1}-\frac{s_{1}}{2}, L_{1}+\frac{s_{1}}{2}\right)\right)$ is a $\Delta$ null subset of $\mathbb{T}$. This contradicts with closedness of $\Gamma_{f}$. That means $L_{1} \leq L_{2}$ and so $L_{1}=L_{2}$.

Theorem 2.6 Let $f: \mathbb{T} \rightarrow \mathbb{R}$ be a $\Delta$-measurable function. The real number $L$ is $\Delta$-limit inferior of the function $f$ if and only if $=\inf \Gamma_{f}$.

Proof It is easily obtained from Proposition 2.2 and Theorem 2.5.

Theorem 2.7 Let $f: \mathbb{T} \rightarrow \mathbb{R}$ be a $\Delta$-measurable function. Then we have $\liminf _{t \rightarrow \infty} f(t) \leq \Delta-\liminf _{t \rightarrow \infty} f(t) \leq$ $\Delta-\limsup _{t \rightarrow \infty} f(t) \leq \limsup _{t \rightarrow \infty} f(t)$.

Proof The case of $\Delta$ - limsup $_{t \rightarrow \infty} f(t)=\infty$ or $\Delta$ - $\liminf _{t \rightarrow \infty} f(t)=-\infty$ are obvious. Now, assume

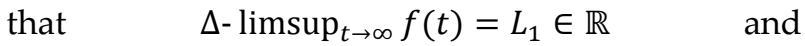
$\Delta$ - $\liminf _{t \rightarrow \infty} f(t)=L_{2} \in \mathbb{R}$. From Theorem 2.3 (ii), for any $s>0$, the set $f^{-1}\left(\left(L_{1}+s, \infty\right)\right)$ is a $\Delta$-null subset of $\mathbb{T}$ then $f^{-1}\left(\left(-\infty, L_{1}+s\right)\right)$ is a $\Delta$-non thin subset of $\mathbb{T}$. From this, we have $L_{2} \leq L_{1}+s$ for all $s>0$. Therefore

$$
\Delta-\liminf _{t \rightarrow \infty} f(t) \leq \Delta-\limsup _{t \rightarrow \infty} f(t) .
$$

Now, first inequality is easily obtained from Proposition 2.2.

Example Let $\mathbb{T}=[0, \infty)$ and $\left(M_{n}\right)$ strictly increasing unbounded sequence in $\mathbb{T}$. Take a sequence $\left(s_{n}\right)$ such that $0<s_{n}<M_{n+1}-M_{n}$ and $s_{n} \rightarrow 0$. One can easily see that $A:=\cup_{n \in \mathbb{N}}\left[M_{n}, M_{n}+s_{n}\right]$ is a $\Delta$-null set. If we define $f: \mathbb{T} \rightarrow \mathbb{R}$

$$
f(t):= \begin{cases}L^{\prime}, & t \in A \\ L, & t \in \mathbb{T}-A\end{cases}
$$

where $L$ and $L^{\prime}$ fixed real numbers such that $L<L^{\prime}$

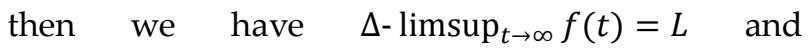
$\limsup _{t \rightarrow \infty} f(t)=L^{\prime}$.

Theorem 2.8 Let $f: \mathbb{T} \rightarrow \mathbb{R}$ be a $\Delta$-bounded function. The function $f$ is $\Delta$-converge to the real number $L$ if and only if 


$$
\Delta-\liminf _{t \rightarrow \infty} f(t)=\Delta-\limsup _{t \rightarrow \infty} f(t) .
$$

Proof If $f$ is $\Delta$-converge to the real number $L$ then $\delta_{\Delta}\left(f^{-1}((L-s, L+s))\right)=1$ for all $s>0$. This implies that both of $f^{-1}((L+s, \infty))$ and $f^{-1}((-\infty, L-$ $s))$ are $\Delta$-null subsets of $\mathbb{T}$ for all $s>0$. By same argument both of $f^{-1}((-\infty, L+s))$ and $f^{-1}((L-$ $s, \infty))$ are $\Delta$-non thin subset of $\mathbb{T}$ for all $s>0$. Therefore from Theorem 2.3 and Theorem 2.4 we have,

$$
\Delta-\liminf _{t \rightarrow \infty} f(t)=\Delta-\limsup _{t \rightarrow \infty} f(t)=L .
$$

Now let $\Delta$ - $\liminf _{t \rightarrow \infty} f(t)=\Delta$ - $\limsup _{t \rightarrow \infty} f(t)=L$. From Theorem 2.3 and Theorem 2.4 we have $\delta_{\Delta}\left(f^{-1}((L-s, L+s))\right)=1$.

\section{References}

[1] Fast, H. Sur La Convergence Statistique. Collog. Math. $1951 ; 2,241-244$.

[2] Fridy, J.A. On Statistical Convergence. Analysis, 1985; 5, 301-313.

[3] Fridy, J.A. Statistical Limit Points. Proc. Amer. Math. Soc. $1993 ; 118,1187-1192$.

[4] Niven, I.; Zuckerman, H. S.; Montgomery, H. An Introduction to the Theory of Numbers. Fifth Ed. Wiley, New York, 1991.

[5] Erdös, P.; Tenenbaum, G. Sur Les Densities de Certaines Suites D'Entries. Proc. London. Math. Soc. 1989; 59, 417-438.

[6] Zygmund, A. Trigonometric Series. 2nd Ed., Cambridge Univ. Press, 1979.

[7] Fridy, J.A.; Orhan, C. Lacunary Statistical Summability. J. Math. Anal. Appl. 1993; 173.

[8] Miller, H.I. A Measure Theoretical Subsequence Characterization of Statistical Convergence. Trans. Amer. Math. Soc. 1995; 347, 1811-1819.

[9] Pehlivan, S.; Mamedov, M. Statistical Cluster Points and Turnpike. Optimization, 2002; 48, 93-106.

[10] Gadjiev, A.D.; Orhan, C. Some Appoximation Theorems Via Statistical Convergence. Rocky Mountain J. Math. 2002; 32, 129-138.

[11] Hilger, S. Ein Maßkettenkalkiil mit Anwendung auf Zentrumsmannigfaltigkeiten. Ph.D. thesis, Universität Würzburg, 1988.

[12] Bohner, M.; Peterson, A. Dynamic Equations on Time Scales. Birkhäuser, Boston, 2001.

[13] Bohner, M.; Peterson, A. Advances in Dynamic Equations on Time Scales. Birkhäuser, Boston, 2003.

[14] Seyyidoğlu, M.S.; Tan, N.Ö. A Note on Statistical Convergence on Time Scale. J. Inequal. Appl.,2012; 219.

[15] Fridy, J.A.; Orhan, C. Statistical Limit Superior and
Limit Inferior. Proc. Amer. Math. Soc. 1997; 125, 3625-3631. [16] Guseinov, G. S. Integration on Time Scales. J. Math. Anal. Appl. 2003; 285, 107-127.

[17] Cabada, A.; Vivero, D. Expression of the Lebesgue $\Delta-$ Integral on Time Scales as a Usual Lebesgue Integral- Application to the Calculus of $\Delta$-Antiderivatives. Math. Comput. Mo-del, 2006; 43, 194-207, 4, 291-310.

[18] Rzezuchowski, T. A Note on Measures on Time Scales. Demonstratio Mathematica, 2005; 38, 179-184.

[19] Deniz, A.; Ufuktepe, U. Lebesgue-Stieltjes Measure on Time Scales. Turk. J. Math. 2009; 33, 27-40.

[20] Seyyidoğlu, M.S.; Tan, N.Ö. On a Generalization of Statistical Cluster and Limit Points Advances in Difference Equations, 2015; 2015:55. 